\title{
The Foundation of the DLR RailwayDynamics Library: the Wheel-Rail-Contact
}

\author{
Andreas Heckmann ${ }^{\diamond}$ Alexander Keck ${ }^{\diamond}$, Ingo Kaiser ${ }^{\diamond}$, Bernhard Kurzeck ${ }^{\diamond}$ \\ ${ }^{\diamond}$ German Aerospace Center (DLR), Institute of System Dynamics and Control \\ Oberpfaffenhofen, 82234 Wessling, Germany \\ andreas.heckmann@dlr.de
}

\begin{abstract}
The formulation of the wheel-rail contact is a crucial issue in simulations considering the running dynamics of railway vehicles. Therefore a modeling environment that is dedicated to railway vehicle dynamics such as the new DLR RaiwayDynamics Library relies on an efficient representation of the kinematics and forces or torques, respectively, that appear at the wheel-rail interface. A number of different formulations have been developed since the underlying rolling contact problem was firstly discussed in literature in 1876. The paper overviews these wheel-rail contact formulations and then presents the implemented variants in detail. The DLR RailwayDynamics Library is used to model and simulate the behavior of an experimental scaled M 1:5 running gear operating on the DLR roller rig. The simulations results are compared and validated with measurements. Keywords: railway vehicles; wheel-rail contact; vehicle dynamics; multibody simulation
\end{abstract}

\section{Literature Review}

\subsection{Introduction}

The wheel-rail interface is a constitutional element of railway vehicles. Knothe et al. [1] tell its three fundamental tasks each associated to a specific contact force component: load-bearing to the vertical force, guiding to the lateral force and traction to the longitudinal force. Although this appears to be very similar to the tire-road interface for automotive vehicles, the wheel-rail interface differs fundamentally due to wheel-rail geometry and the different material behavior of both contact partners which are made of steel.

The contact between wheel and rail in normal

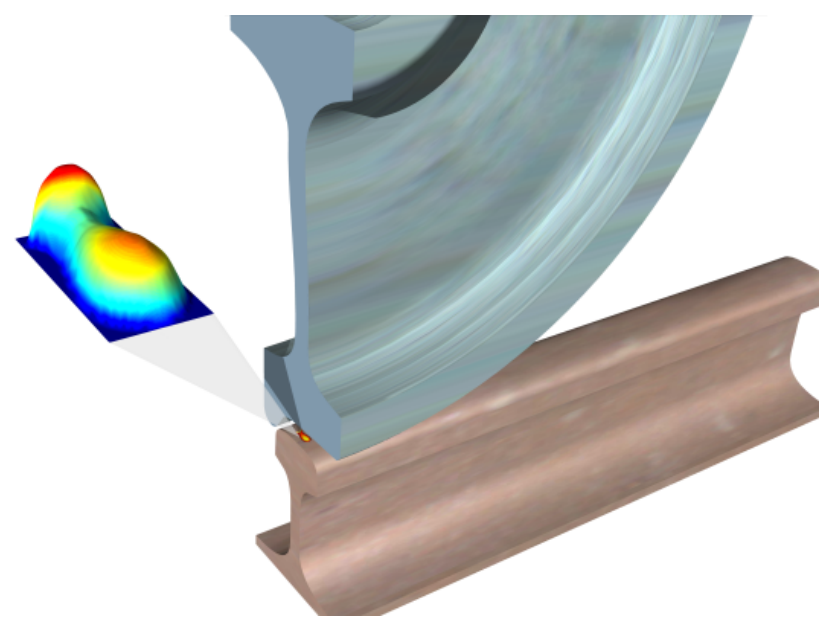

Figure 1: Exemplary contact patch between wheel and rail and a qualitative surface plot of the associated normal stress distribution

direction is very stiff. The deformations of the contact partners add up to $1 / 10$ or $2 / 10 \mathrm{~mm}$, the contact area has an approximate size of 1 to $2 \mathrm{~cm}^{2}$ although the usual transmitted vertical loads are very high, i.e. in the order of magnitude of 10 tons. Fig. 1 gives an impression of the size of the contact patch and the normal stresses here presented with a maximum of roughly $700 \mathrm{~N} / \mathrm{mm}^{2}$.

In the tangential direction the contact behavior is ruled by friction. Therefore it depends on the normal contact conditions with friction coefficients between 0.1 and 0.4 and exhibits non-linear behavior and saturation.

This general specification already exposes the complexity of the wheel-rail contact problem which is also demonstrated by Fig. 2. There, the contours visualize the normal stress distribution as it is already given by the surface plot in Fig. 1 . The arrows represent the tangential stress vectors and the circles indicate the points where the wheel surface slips along the rail surface since adhesion 


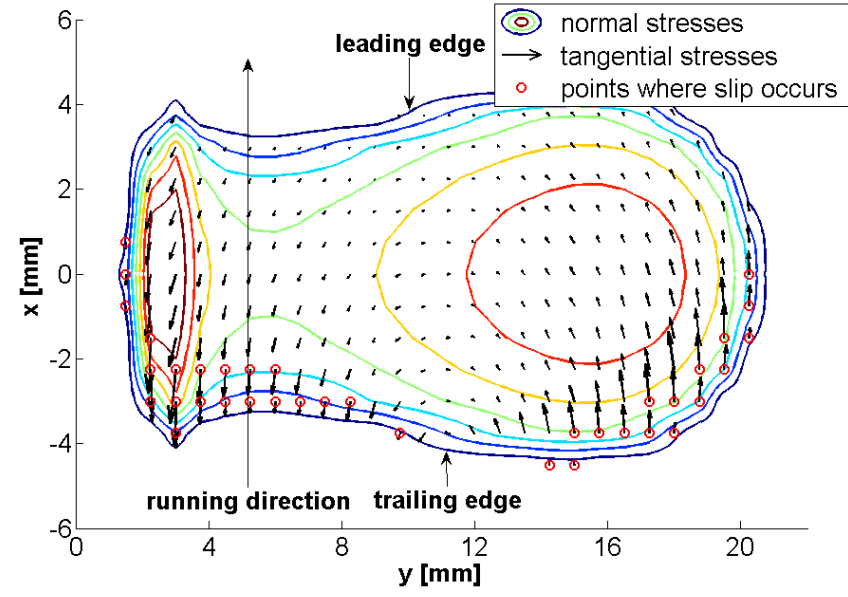

Figure 2: Exemplary contact stresses and the associated slip region evaluated according to Kalker's non-elliptical rolling contact theory [2]

is depleted locally.

The fundamental normal and tangential contact properties motivate the use of conical wheel profiles so that the normal contact does not only carry vertical loads but as well contributes to the lateral guiding task. The additional consideration of undesired profile changes due to wear led to the evolution of specific wheel and rail profiles as they are shown in Fig. 3, see [3] for a more elaborate discussion on the significance of the profile geometry. It is obvious that such profile design introduces another complexity into the formulation of a wheel-rail contact model due to the geometry. For example, the shape of the outer stress field boundary in Fig. 2, usually denoted as non-elliptical, is a particular result introduced by the non-linear profile geometry.

\subsection{Basic Modeling Issues}

The goal of the modeling presented in this paper is to provide the capability of simulating the running dynamics of railway vehicles. Hence, it is important that the resultant forces that dominate the motion of the railway vehicle and are to be computed very often during one simulation job, are evaluated in reasonable accuracy and with low computational burden. However, it is not intended to give detailed and high accuracy information on the contact stress distribution as shown Fig. 2 and as it is required in order to examine e.g. rolling contact fatigue.

It is therefore a usual approach to review the

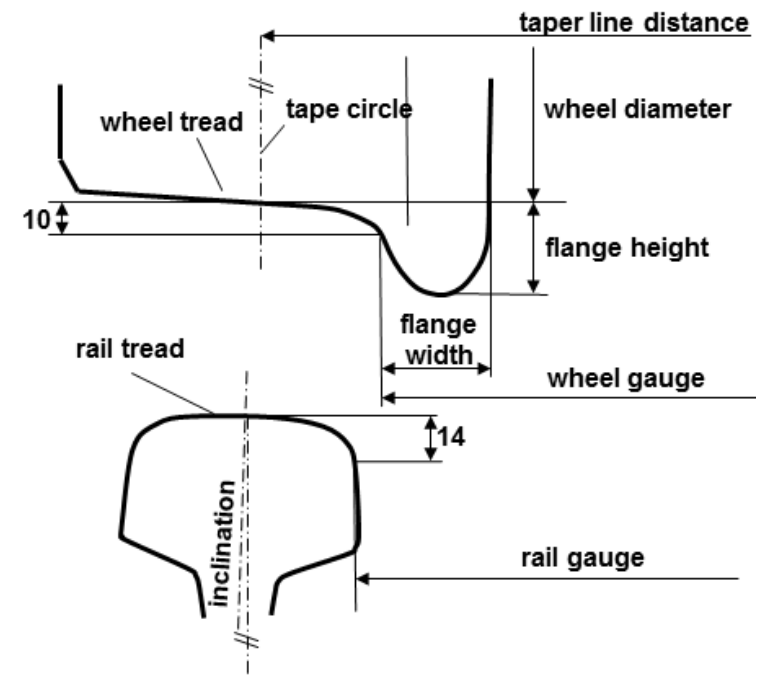

Figure 3: Terminology and characteristics of wheel and rail profile

wheel-rail contact problem looking for reasonable assumptions that simplify the modeling and reduce the computational effort. Tab. 1 gives an overview on widespread assumptions which may be applied according to the specific analysis goals at hand.

The separation assumption for example allows for introducing a virtual contact point where the resultant forces and torques acting on the wheel are assumed to be attached in order to simulate running dynamics. However the magnitude of the forces and torques are evaluated considering the contact problem separately.

Presuming identical materials for wheel and rail, the separation assumption together with the halfspace approximation makes it possible to evaluate the normal contact first and independently and then derive the tangential stress quantities on top of it [1, Sec. 3.3.2].

Combining these two main simplification ideas with the discussion in Sec. 1.1 facilitates the formulation of the wheel-rail rolling contact problem by subdividing it into the following three subtasks that may be solved subsequently: the geometrical problem, the normal contact problem and the tangential contact problem.

A basic modeling aspect that is related to the multibody representation of the wheel-rail contact is the distinction drawn between rigid and elastic contact. In multibody theory the latter is equivalent to the description of the normal wheel-rail contact as a force law, while the ideal rigid contact leads to the concept of a kinematical constraint, so 


\begin{tabular}{ll}
\hline Separation assumption & $\begin{array}{l}\text { The deformations of the contacting bodies do not influence their over- } \\
\text { all motion so that wheel and rail may be assumed to be rigid bodies. } \\
\text { Structural and contact mechanics may be considered independently from } \\
\text { each other. }\end{array}$ \\
\hline Linearity & $\begin{array}{l}\text { The deformation fields are geometrically linear. } \\
\text { Half-space assumption } \\
\text { The contact area is very small compared to the characteristic dimensions } \\
\text { of the related bodies in contact. }\end{array}$ \\
\hline Ideal material & The material is linear-elastic, homogeneous and isotropic. \\
\hline Identical material & Wheel and rail consist of identical materials. \\
\hline Steady state assumption & $\begin{array}{l}\text { The running velocity is small compared to the wave propagation within } \\
\text { the material, so that quasi-static conditions are valid with respect to the } \\
\text { contact problem. }\end{array}$ \\
\hline Hertz assumption & $\begin{array}{l}\text { The geometry of the contacting surfaces may be approximated as elliptic } \\
\text { paraboloids so that the contact patch turns out to be a plain ellipse. }\end{array}$ \\
\hline Dry Coloumb friction & $\begin{array}{l}\text { Only dry friction is considered. The friction coefficient is constant and } \\
\text { valid for adhesion as well as for sliding. }\end{array}$ \\
\hline
\end{tabular}

Table 1: Overview on assumptions frequently exploited for wheel-rail contact modeling, cp. [1, Ch. 3]

that the equations of motion form a differentialalgebraic system [4]. The rigid contact requires a sophisticated preprocessing to guarantee the differentiability of the constraint equation and avoid artificial contact point jumps [5]. However, it is assumed to need less computational effort for time integration compared to the elastic contact that introduces very high stiffnesses into the equations of motion, but provides a more general applicability [6].

In the discussion so far it is assumed that the contact between wheel and rail forms one continuous contact area that may be idealized by one single contact point, which in fact is the dominant standard case. However specific configurations such as switch crossing or light urban and metro railway vehicles in sharp curves exist where multiple, non-connected contact areas between wheel and rail surface occur. These configurations require the consideration of multiple contact points, see e.g. [7] or [4].

\subsection{The Normal Contact Problem}

The basis for the highly accurate non-elliptical contact description e.g. shown in Fig. 2 has been set by Kalker [8] who implemented the program Contact that became a reference for railway contact problems. Contact fully accounts for the profile geometry of wheel and rail so that the simplification of the Hertz assumption from Tab. 1 is not employed. However, the half-space assumption is exploited to evaluate the stress and deforma- tion field numerically. Contact is mainly applied for verification purposes in offline calculations. In addition, the same methodology has been used for detailed research about the influence of structural dynamics of wheel and rail on the vehicletrack interaction [2]. However for industrial applications, the accurate consideration of the nonelliptical contact requires too many computational resources, see e.g. [9] for optional approximations.

A very frequently used normal contact model in railway dynamics analysis is the elliptical contact. Here, the deviation of the contacting surfaces of wheel and rail from the ideal ellipsoidal shape is neglected and the contact stresses and deformations may be evaluated analytically according to the Hertz theory, see e.g. [10, Sec. 4.II.A].

For the sake of completeness the Finite Element Method shall be mentioned as a very general way to evaluate contact problems that does not rely on any of the mentioned simplifications in Tab. 1. Effects such as surface hardening, material flows or damage mechanism can be taken into account, see e.g. [11].

\subsection{The Tangential Contact Problem}

As soon as a relative motion, the so-called slip, between the contact point on the wheel and its counterpart on the rail occurs, tangential forces are transmitted. The program Contact is a widespread accepted reference to solve as well this tangential contact problem.

However in order to facilitate vehicle simu- 
lations, Kalker proposed a simplified theory of rolling contact based on discretized ellipses and provided the Fastsim [12] algorithm that nevertheless takes traction and saturation into account.

For vanishing slip, if e.g. only very small traction forces are given, the linearized theory of Kalker [8, Sec. 2.2.2] is valid. Here, the tangential forces are a linear function of the slip.

A different class of tangential contact models try to get along with purely analytical considerations, as it is already done for the normal contact using the Hertz solution. However, these contact models require an assumption concerning the shape of the stick and slip region within the contact area. The basic idea originates from Carter [13], who showed that the tangential stress distribution of a cylindrical body rolling on a plane may be presented by two nested ellipses. The extension to three dimensions however relies on approximations that were proposed e.g. by Vermeulen and Johnson or by Shen, Hedrick and Elkins [13].

Following the same basic concept Polach [14] published a very efficient tangential contact solution in 1992 that coincides with Kalker's linear theory in the case of vanishing slip. In addition an extension is proposed for applications on the adhesion limit in which high traction forces are involved [15]. Besides the Fastsim algorithm the Polach model is wide-spread for multibody railway vehicle simulations today, see [16] for an assessment of various approaches.

\subsection{Review Conclusions}

The Modelica implementation of the wheel-rail contact is in particular supposed to support the development of new railway vehicle control concepts that are on the agenda of the DLR internal project Next Generation Train (NGT) [17]. For this purpose accuracy and computational effort have to be compromised. Therefore it has been decided to implement a rigid elliptical single point contact with tangential force law according to Polach.

\section{Theory}

\subsection{Profile Geometry and Contact}

In order to formulate the rigid contact, we look for an implicit constraint equation that defines the vertical wheel displacement $z_{w}$ as a function of the lateral displacement $y_{w}$, the yaw angle $\psi$ and the

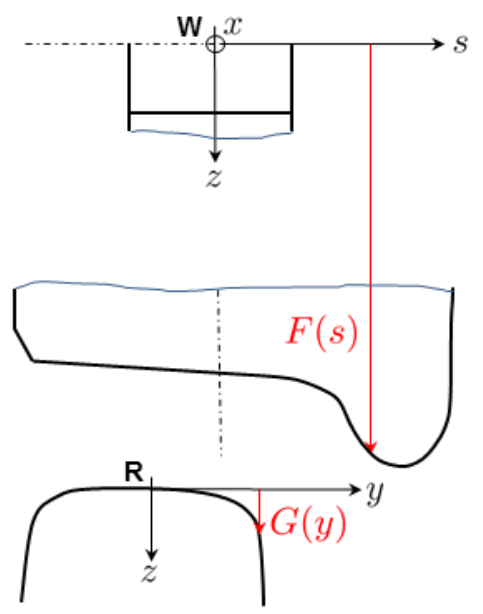

Figure 4: Parametrization of the profile geometry.

roll angle $\varphi$ of the wheel [5], i.e.

$$
z_{w}+f\left(y_{w}, \varphi, \psi\right)=0
$$

which is two times continuously differentiable [18]. $z_{w}$ and $y_{w}$ are resolved with respect to the rail coordinate system $\mathrm{R}$ in Fig. 4, where the parametrization of the wheel profile $F=F(s)$ and the rail profile $G=G(y)$ is visualized as well.

The wheel surface $S_{w}$ may be given in cylindrical coordinates of the wheel, i.e. $S_{w}:(F(s), \tau, s)^{T}$, or resolved with respect to $R$ :

$$
S_{w}: \boldsymbol{c}_{S}=\left(\begin{array}{c}
0 \\
y_{w} \\
z_{w}
\end{array}\right)+A(\varphi, \psi)\left(\begin{array}{c}
F(s) \sin \tau \\
s \\
F(s) \cos \tau
\end{array}\right),
$$

where $A(\varphi, \psi)$ represents the rotations around the $x$ - and the $z$-axis of the wheel.

Those points on $S_{w}$, whose normal vectors to the surface are parallel to the $(y, z)$-plane of the rail define the curve $C_{w}$ that is the projection of the wheel contour in the $(y, z)$-plane of the rail [19, Sec. 2.2]:

$$
\begin{array}{r}
C_{w}: \boldsymbol{c}_{C}=\left\{\boldsymbol{c}_{S}: \sin \tau(s)=-\tan \psi F(s)\right. \\
\left.\cos \tau(s)=\sqrt{1-\sin ^{2} \tau(s)}\right\} .
\end{array}
$$

We now specify the curve $C_{E}$ to be independent from the vertical position of the wheel $z_{w}$

$$
C_{E}: \boldsymbol{c}_{E}=\left(x_{E}, y_{E}, z_{E}\right)^{T}:=\boldsymbol{c}_{C}-\left(0,0, z_{w}\right)^{T}
$$

which can be exploited to relate the wheel contour $z_{E}=z_{E}\left(s ; y_{w}, \varphi, \psi\right)$ to the associated rail profile 
$G\left(y_{E}\right)=G\left(s ; y_{w}, \varphi, \psi\right)$ as a function of the parameter $s, \underline{s} \leq s \leq \bar{s}$.

Wheel and rail are in contact iff

$$
z_{w}+\max _{\underline{s} \leq s \leq \bar{s}} \underbrace{\left(z_{E}-G\right)}_{\Delta\left(s ; y_{w}, \varphi, \psi\right)}=0 .
$$

The function $\Delta\left(s ; y_{w}, \varphi, \psi\right)$ in (5) is called the height function [5]. The value $s^{*}$ that is assigned to the global maximum of $\Delta$ specifies the contact point.

The direct application of (5) as constraint equation in multibody simulation for standard wheelrail profiles such as S1002 and UIC60 does not make sense, since these profiles expose abrupt curvature changes and promote artificial jumps of the normal contact forces [4]. Therefore Arnold et al. [5] propose to use a regularization parameter $\alpha>0$ in the range $10^{-5} \ldots 5 \cdot 10^{-5}$ in the following way:

$$
\begin{gathered}
z_{w}+\operatorname{smax}_{s}^{(\alpha)} \Delta\left(s, y_{w}, \varphi, \psi\right)=0, \\
\operatorname{smax}_{s}^{(\alpha)} \Delta:=\alpha \ln \frac{\int_{\underline{s}}^{\bar{s}} \exp \left(\frac{\Delta\left(s, y_{w}, \varphi, \psi\right)}{\alpha}\right) \mathrm{d} s}{\int_{\underline{s}}^{\bar{s}} \mathrm{~d} s} .
\end{gathered}
$$

For small values of $\alpha$ it can be shown that $\operatorname{smax}_{s}^{(\alpha)} \Delta \leq \max _{s} \Delta$, i.e. (6) yields values of $z_{w}$ that represent a small penetration $\delta_{r w}$ of the wheel and rail bodies. The proposed values of $\alpha$ are chosen in such a way that $\delta_{r w}$ corresponds to the physical deformations of the contact partners, which could be evaluated e.g. according to the Hertzian theory. Therefore (6) is called the quasi-elastic contact model by Arnold et al..

The listing below shows that the geometrical problem is tackled by a Modelica function that takes $y_{w}, \varphi, \psi$ and $\alpha$ as inputs and mainly returns $z_{w}$ in addition to quantities that are necessary for the tangential contact evaluation:

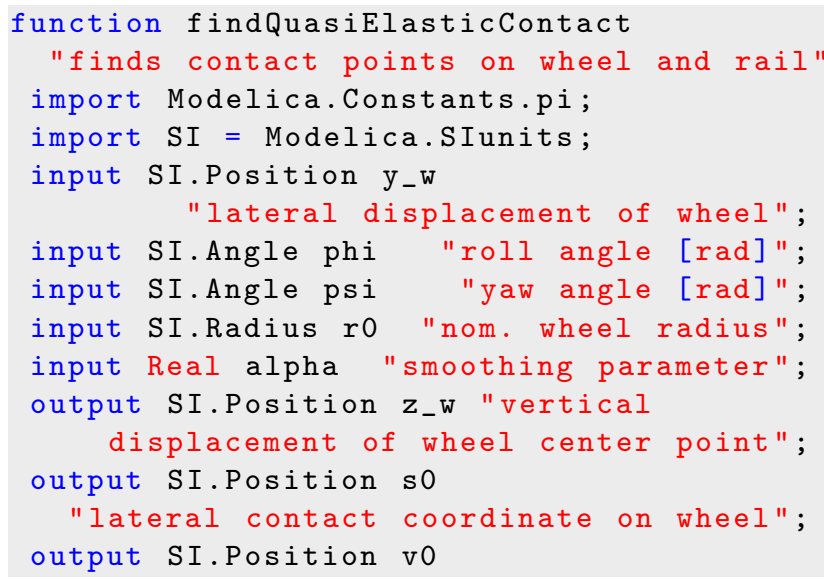

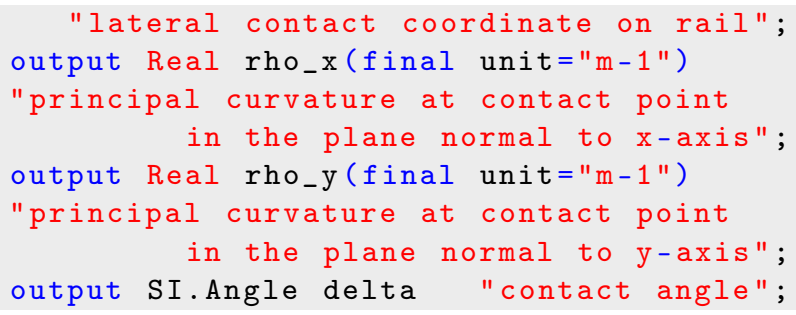

The quantity $s$ is an internal vector variable of this function which disretizes the wheel profile, e.g. $s=\{-0.05,-0.0499, \ldots ., 0.05\}$.

\subsection{Kinematics}

Consider the coordinate system in Fig. 5 in order to resolve the vectorial quantities in what follows. The $\boldsymbol{e}_{z}$-vector is normal to the wheel and the rail surface in the contact point $\mathrm{C}, \boldsymbol{e}_{x}$ is perpendicular to the wheel axis and heads in running direction. $v_{0}$ is that component of the absolute velocity of the wheel center point $\boldsymbol{v}_{w}$ that points in $\boldsymbol{e}_{x}$-direction. $\boldsymbol{\omega}_{w}$ is the absolute angular velocity of the wheel that includes yaw, roll and the overturning motion $\omega_{0}$ of the wheel.

The sliding velocity $\boldsymbol{v}_{s}$ [20, Sec.2.6.2] in C follows from

$$
\boldsymbol{v}_{s}=\boldsymbol{v}_{w}+\boldsymbol{\omega}_{w} \times \boldsymbol{r}_{C}
$$

and is used together with $\boldsymbol{\omega}_{w}$ to compose the slip vector $\boldsymbol{\nu}$ with the longitudinal slip $\nu_{x}$, the lateral

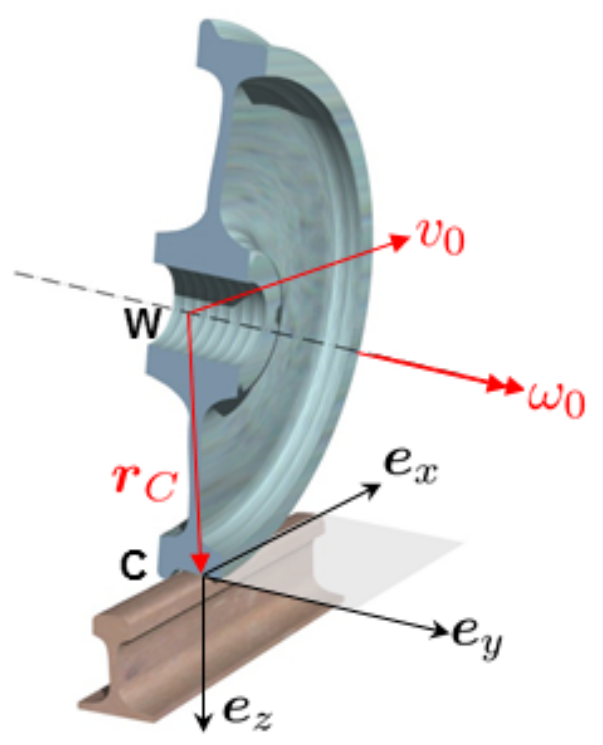

Figure 5: Coordinate system associated to the plane through contact point $\mathrm{C}$. 


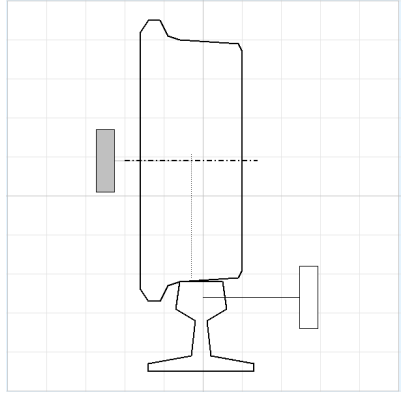

Figure 6: Icon layer of the contact model.

slip $\nu_{y}$ and the spin $\phi_{z}$ :

$$
\boldsymbol{\nu}:=\left(\begin{array}{c}
\nu_{x} \\
\nu_{y} \\
\phi_{z}
\end{array}\right)=\frac{1}{v_{0}}\left(\begin{array}{c}
v_{s x} \\
v_{s y} \\
\omega_{w z}
\end{array}\right)
$$

Fig. 6 shows the the icon layer of the contact model with two multibody frame connectors from the Modelica Standard Library. The frames have to be connected to the center of the wheel and to the reference point on the rail which correspond to the points $\mathrm{W}$ and $\mathrm{R}$ in Fig. 4. The kinematical information of these two frames are required to evaluate (7) and (8).

The contact model does not have a state but represents a loop-closing element, which corresponds to the property that the wheel surface is constrained to be in touch with the rail surface. The constraint is formulated using the function from Sec. 2.1. As a result, the contact force in normal direction, i.e. normal to the plane through the point $\mathrm{C}$ according to Fig. 5 implicitly follows from the closed loop condition.

\subsection{Hertzian Normal Contact}

The pressure distribution $p$ in the contact plane according to the Hertzian theory [21, (3.65)] is given by

$$
p(x, y)=\frac{3 f_{n}}{2 \pi a b} \sqrt{1-\frac{1}{a^{2}}-\frac{1}{b^{2}}},
$$

where $f_{n}$ represents the absolute value of the normal force, while $\mathrm{a}$ and $\mathrm{b}$ denote the semi-axes of the contact ellipse and follow from

$$
a=m \sqrt[3]{\frac{3\left(1-\kappa^{2}\right) f_{n}}{E(A+B)}}, b=n \sqrt[3]{\frac{3\left(1-\kappa^{2}\right) f_{n}}{E(A+B)}} .
$$

Beside Young's modulus $E$ and Poisson's ratio $\kappa$ that are assumed to be identical for wheel and rail, (10) uses auxiliary terms that are determined by the curvature of the wheel $\rho_{w x}$ and the rail $\rho_{r x}$ measured in the plane normal to the $x$-axis and the wheel curvature in the plane normal to the $y$-axis $\rho_{w y}$ :

$$
A=\rho_{w x}+\rho_{r x}, \quad B=\rho_{w y}, \quad \vartheta=\arccos \frac{A-B}{A+B} .
$$

$m$ and $n$ are coefficients depending on elliptical integrals and specify the eccentricity of the contact ellipse. Tab. 2 is an extraction of [21, Tab. 3.4] to give an impression on their quantitative values.

\begin{tabular}{c|c|c|c|c|c|c}
$\vartheta$ & $0^{\circ}$ & $0.5^{\circ}$ & $1^{\circ}$ & $10^{\circ}$ & $45^{\circ}$ & $90^{\circ}$ \\
\hline \hline $\mathrm{m}$ & $\infty$ & 61.4 & 36.89 & 6.604 & 1.926 & 1 \\
\hline $\mathrm{n}$ & 0 & 0.1018 & 0.1314 & 0.3112 & 0.604 & 1
\end{tabular}

Table 2: Hertzian parameters for the contact ellipse.

In [5], it is proposed to introduce a weigthed mean value of the curvatures in (10) that corresponds to (6), e.g. to consider the wheel curvature

$$
\bar{\rho}_{w y}:=\frac{\int_{\underline{s}}^{\bar{s}} \rho_{w y}(s) \exp \left(\frac{\Delta}{\alpha}\right) \mathrm{d} s}{\int_{\underline{s}}^{\bar{s}} \exp \left(\frac{\Delta}{\alpha}\right) \mathrm{d} s} .
$$

Eq. (11) is implemented in the function presented in Sec. 2.1 as indicated by the output values given there.

\subsection{Linear Tangential Contact}

For vanishing slip Kalker's, i.e. for small values of $\boldsymbol{\nu}$, linear theory [8, Sec. 2.2.2] is valid so that the creep forces $f_{x}$ and $f_{y}$ in and the torque $l_{z}$ normal to the $x y$-plane depend linearly on the slip $\boldsymbol{\nu}$ under consideration of the shear modulus $G$ :

$$
\begin{gathered}
\boldsymbol{f}=\left(\begin{array}{l}
f_{x} \\
f_{y} \\
l_{z}
\end{array}\right)=\boldsymbol{C} \boldsymbol{\nu} \\
\boldsymbol{C}=-a b G\left(\begin{array}{ccc}
C_{11} & \text { with } \\
0 & C_{22} & \sqrt{a b} C_{23} \\
0 & -\sqrt{a b} C_{23} & a b C_{33}
\end{array}\right) .
\end{gathered}
$$

For the sake of demonstration Tab. 3 shows exemplary values of the coefficients that appear in (12).

\subsection{Polach's Tangential Contact}

According to Polach [14] the torque $l_{z}$ in (12) is usually very small and can be neglected, while the 


\begin{tabular}{c|c|c|c|c|c|c}
$b / a$ & 1 & 0.8 & 0.6 & 0.4 & 0.2 & 0.1 \\
\hline \hline$C_{11}$ & 4.12 & 4.36 & 4.78 & 5.57 & 7.78 & 11.7 \\
\hline$C_{22}$ & 3.67 & 3.99 & 4.5 & 5.48 & 8.14 & 12.8 \\
\hline$C_{23}$ & 1.47 & 1.75 & 2.23 & 3.24 & 6.63 & 14.6 \\
\hline$C_{33}$ & 1.19 & 1.04 & 0.892 & 0.747 & 0.601 & 0.526
\end{tabular}

Table 3: Coefficients of Kalker's linear theory of rolling contact for $\kappa=0.25$ and $a>b$ [8, E3].

influence of the spin $\phi_{z}$ on the lateral creep force $f_{y}$ may be of considerable importance. This is why his creep force law considers two lateral components: $f_{y}^{\diamond}$ denotes the creep force that originates from lateral slip and $f_{y}^{*}$ is associated to the spin:

$$
\boldsymbol{f}=\left(\begin{array}{c}
f_{x} \\
f_{y}^{\diamond}+f_{y}^{*} \\
0
\end{array}\right)
$$

The tangential force in Polach's model is defined in the direction of the slip resultant $\bar{\nu}$ taking the influence of the spin into account:

$$
\begin{gathered}
\bar{\nu}=\sqrt{\nu_{x}^{2}+\bar{\nu}_{y}^{2}}, \\
\bar{\nu}_{y}=\left\{\begin{array}{lll}
\nu_{y}+a \phi_{z} & \forall & \left|\nu_{y}+a \phi_{z}\right|>\left|\nu_{y}\right| \\
\nu_{y} & \forall & \left|\nu_{y}+a \phi_{z}\right| \leq\left|\nu_{y}\right|
\end{array} .\right.
\end{gathered}
$$

In order to evaluate the resulting friction force $\bar{f}$ it is postulated that the tangential stresses grow linearly with the distance from the leading edge until saturation is reached. Hence, the analytical integration of the assumed stress field over the contact patch leads to

$$
\bar{f}=-\frac{2 f_{n} \mu}{\pi}\left(\frac{k_{a} \varepsilon}{1+\left(k_{a} \varepsilon\right)^{2}}+\arctan \left(k_{s} \varepsilon\right)\right),
$$

where $\mu$ denotes the friction coefficient, $k_{a}$ and $k_{s}$, $k_{s} \leq k_{a} \leq 1$, are reduction factors associated to the adhesion or the slip area, respectively. They have been introduced by Polach [15] in order to account for wet or polluted conditions. $\varepsilon$ represents the gradient of the tangential stress in the area of adhesion at the leading edge of the contact patch and is related to the coefficients of Kalker's linear theory:

$$
\varepsilon=\frac{G \pi a b C_{j j}}{4 f_{n} \mu}, \quad C_{j j}=\sqrt{\frac{C_{11}^{2} \nu_{x}^{2}}{\nu_{x}^{2}+\nu_{y}^{2}}+\frac{C_{22}^{2} \nu_{y}^{2}}{\nu_{x}^{2}+\nu_{y}^{2}}} .
$$

The subdivision of the $\bar{f}$ into its two components corresponds to the slip partitions:

$$
f_{x}=\bar{f} \frac{\nu_{x}}{\bar{\nu}}, \quad f_{y}^{\diamond}=\bar{f} \frac{\nu_{y}}{\bar{\nu}} .
$$

The remaining creep force component $f_{y}^{*}$ is evaluated separately yielding

$$
\begin{gathered}
\left.f_{y}^{*}=-\frac{\phi_{z}}{\bar{\nu}} \frac{9 a f_{n} \mu\left(1+6.3\left(1-e^{-\frac{a}{b}}\right)\right)}{16}\right) \cdot \\
\cdot\left[\varepsilon^{*}\left(\frac{\delta^{3}}{-3}+\frac{\delta^{2}}{2}-\frac{1}{6}\right)+\frac{\sqrt{\left(1-\delta^{2}\right)^{3}}}{3}\right],
\end{gathered}
$$

where the tangential stress gradient due to spin $\varepsilon^{*}$ and the abbreviation $\delta$ are used:

$\varepsilon^{*}=\frac{8 G b \sqrt{a b} k_{a} C_{23}}{3 f_{n} \mu\left(1+6.3\left(1-e^{-\frac{a}{b}}\right)\right)}\left|\bar{\nu}_{y}\right|, \delta=\frac{\left(\varepsilon^{*}\right)^{2}-1}{\left(\varepsilon^{*}\right)^{2}+1}$.

For traction vehicles running on adhesion limit, the dependence of the friction coefficient on the slip velocity may be considered relating the maximum friction coefficient $\mu_{0}$ to the limit friction coefficient $\mu_{\infty}$ at infinite slip velocity introducing the parameter $B$ :

$$
\mu=\mu_{0}\left[\left(1-\frac{\mu_{\infty}}{\mu_{0}}\right) e^{-B v_{s x}}+\frac{\mu_{\infty}}{\mu_{0}}\right]
$$

The above used constants $k_{a}, k_{s}, \mu_{0}, \mu_{\infty}$ and $B$ are heuristic quantities that have been introduced to account for deviations from theory observed by measurements. Tab. 4 quotes Polach to give typical values.

\begin{tabular}{c|c|c|c|c|c} 
conditions & $k_{a}$ & $k_{s}$ & $\mu_{0}$ & $\mu_{\infty}$ & $B[\mathrm{~s} / \mathrm{m}]$ \\
\hline \hline dry & 1.00 & 0.40 & 0.55 & 0.22 & 0.60 \\
\hline wet & 0.30 & 0.1 & 0.30 & 0.12 & 0.20
\end{tabular}

Table 4: Typical model parameters for dry and wet conditions of the real wheel-rail contact [15].

\section{Application}

\subsection{Project Background}

Fig. 7 shows an experimental running gear in scale 1:5 that operates on the DLR roller rig in Oberpfaffenhofen. Unlike usual wheel-set configurations this running gear has independently rotating wheels each driven by one wheel hub motor. Two opposite front wheels are mounted together on a cranked beam, the two rear wheels mounted on their carrier constitute the identical second wheel pair unit. Each wheel pair unit is connected to the central frame having one rotational degree of freedom around the vertical axis. 


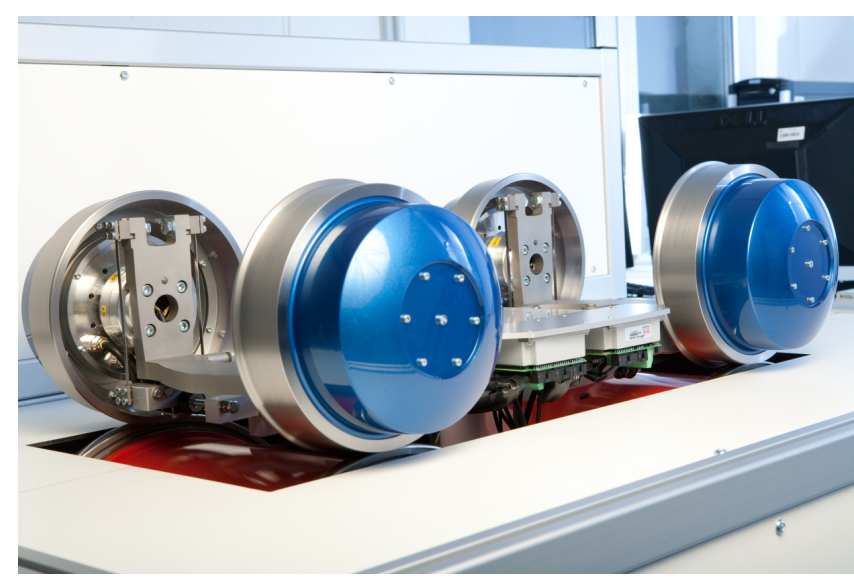

Figure 7: M 1:5 roller rig of DLR with experimental running gear for mechatronic guidance research.

That way the wheel pair units may perform independent yaw motions relative to the central frame. The central frame has two degrees of freedom with respect to the roller rig basis so it may move laterally and yaw.

The running gear has been designed in order to develop a new mechatronic guidance concept that allows for active steering. Significant wear, noise and weight reduction together with benefits that result from the low-floor configuration are goals that are on the agenda of the DLR internal project NGT [17].

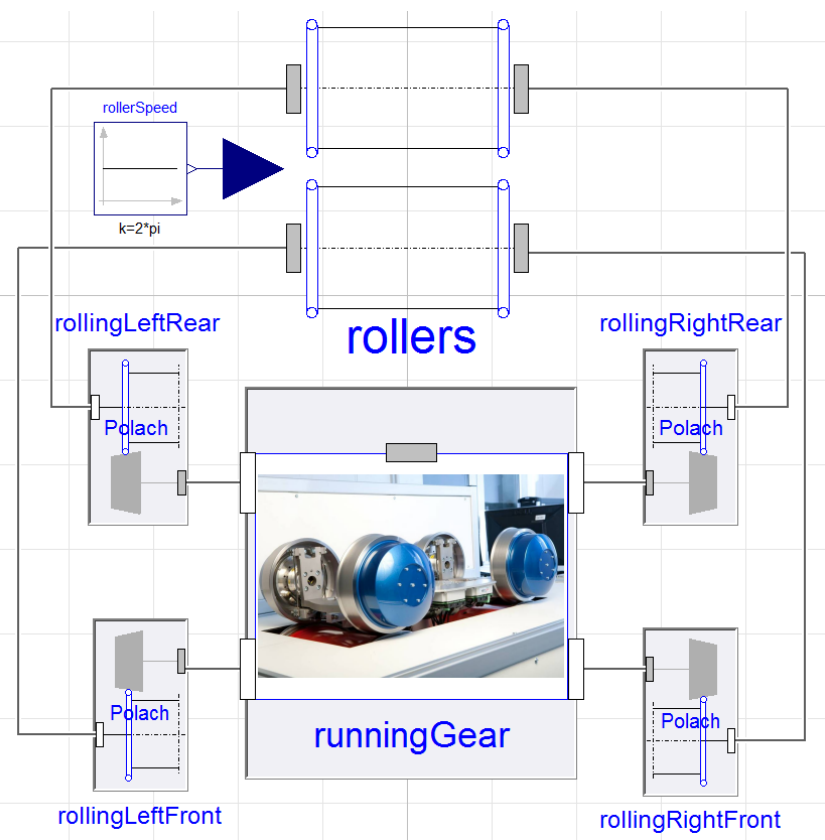

Figure 8: Diagram layer of the running gear model on the roller rig.

\subsection{Model Particularities}

In parallel to the experimental environment a simulation model of the running gear on the test rig has been established in Modelica. The diagram layer of the Modelica model is visualized in Fig. 8 while an animation of the running gear operating on the roller rig is presented in Fig. 9. Both environments together are supposed to support the development of advanced control design concepts.

However the aim of this paper is rather the modeling and the validation of the wheel-rail contact that has been introduced in Sec. 2 and is instantiated four times in Fig. 8. The contact model named Polach uses two multibody frame connectors to be linked to the center of the wheel and to the center of the associated roller. Since the contact model represents a loop-closing element, the contact force in normal direction, implicitly follows from the closed loop condition. The tangential forces are evaluated as described in Sec. 2.5.

Fig. 10 shows the main part of the menu used to parametrize the contact model. The wheel profile here is not a standard one, but can here be represented by a true conical shape. The control of the running gear is defined in such a way that only a repective region of the wheel tread is in contact. Therefore the parameter $r_{-}$wheel_FrontView is set to $1 \cdot 10^{12}$ or infinity, respectivley in Fig. 10.

The discussion in Sec. 2.1 is implicitly restricted to the contact of wheels to prismatic rails, which is different here due the geometry of the rollers with

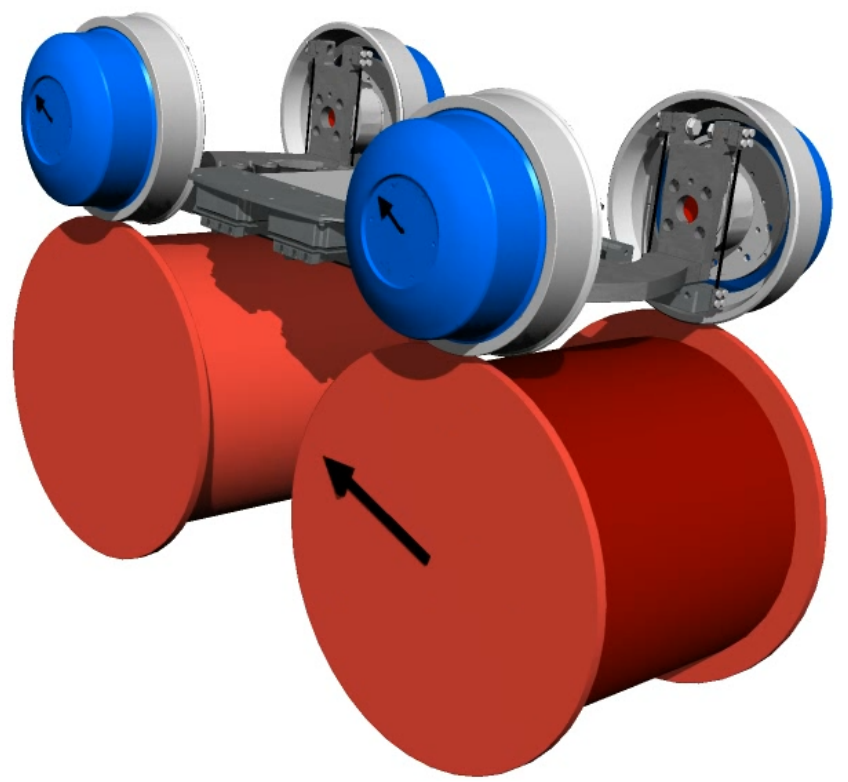

Figure 9: Animation of the running gear model. 


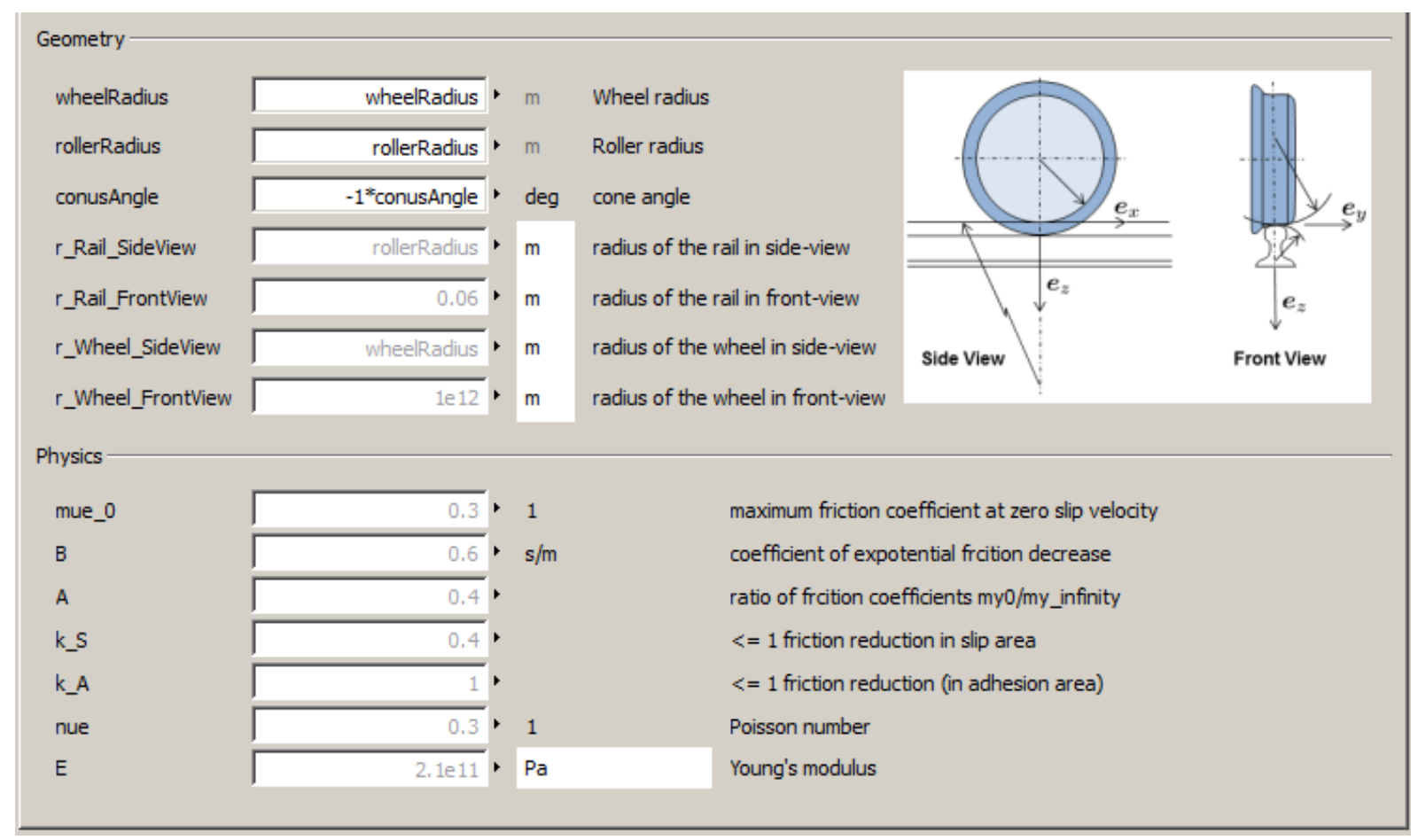

Figure 10: Parameter menu of the wheel-rail contact for conical wheel and curved roller profiles.

$0.18 \mathrm{~m}$ radius. As soon as a wheel unit performs a yawing motion the two wheels leave the apexes of the associated rollers and slightly run downhill. This behavior is considered in the model but has been disregarded in Sec. 2, see [19, Sec. 2.2.2].

\section{$3.3 \quad$ Results}

A feature of the running gear are the force-torque sensors, that are assembled at the bearing of each wheel. Therefore the capability is given to compare simulation and measurement results with particular respect to the wheel-rail forces.

Fig. 11 to Fig. 13 show wheel-rail forces at the rear wheel on the right hand side. After the measurements have been low-pass filtered using a cutoff frequency of $20 \mathrm{~Hz}$, the rotation frequencies of the roller and wheels still show up clearly, so that two narrow frequency bands of $0.2 \mathrm{~Hz}$ around these frequencies have been filtered out additionally. It is a current work field to eliminate or at least reduce the influence of the related disturbance sources.

The control of the running gear is set up in such a way that the running gear performs an artificial so-called hunting motion with $0.5 \mathrm{~Hz}$ frequency and a lateral amplitude of $8 \mathrm{~mm}$. Whenever a conventional wheel-set is laterally excited e.g. by rail irregularities its dynamical response is a lateral oscillation calling hunting. As along as the running velocity does not exceed a certain level, this motion is asymptotically stable. This is a desired dynamical property and a specific aspect of the wheel-rail profile design mentioned in Sec. 1.1. In addition hunting promotes wear not to be locally concentrated but distributed over a larger region of the wheel surface.

However independently rotating wheels do not show this passive behavior so that it has to be in-

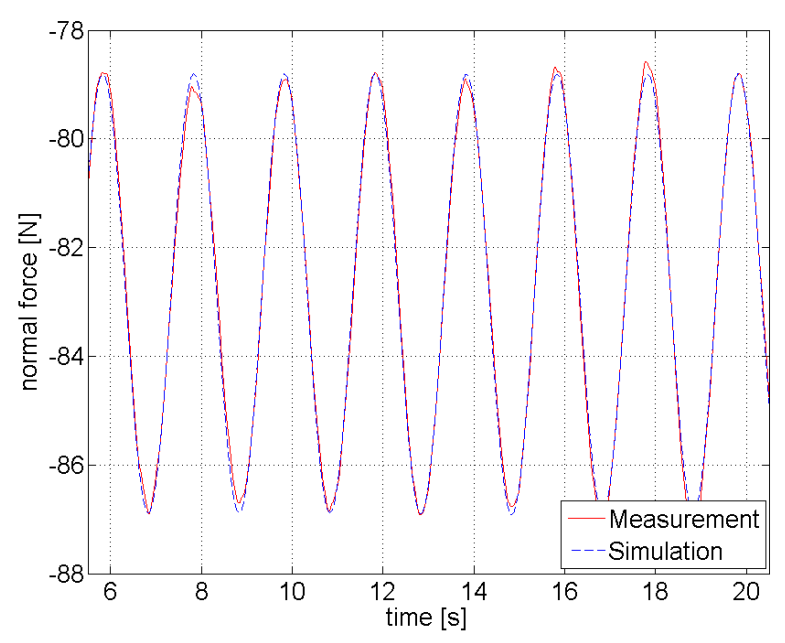

Figure 11: Comparison of normal wheel-rail force, $v_{0}=6 \mathrm{~m} / \mathrm{s}, 0.5 \mathrm{~Hz}$ hunting frequency. 


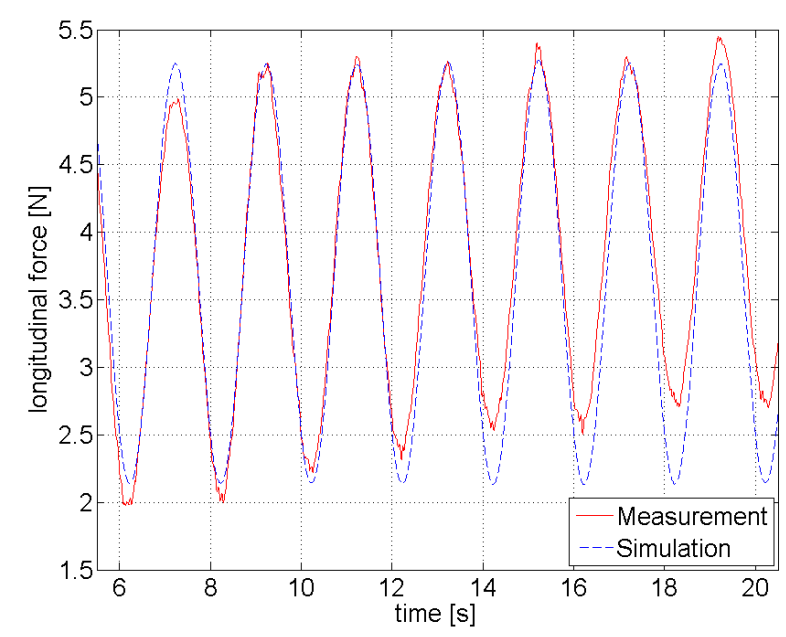

Figure 12: Comparison of longitudinal wheel-rail force, $v_{0}=6 \mathrm{~m} / \mathrm{s}, 0.5 \mathrm{~Hz}$ hunting frequency.

troduced by feed-back control. The artificial hunting by control is the first proof a new mechatronic running gear concept has to stand, i.e. the mechatronic running gear has to perform at least as good as the conventional wheel-set design, before additional benefits could be approached.

Due to the hunting the normal wheel-rail force in Fig. 11 oscillates between 79 and 87 N. Measurements and simulation results corrrepond very good. The longitudinal forces in Fig. 12 show a long-wave deviation but are nevertheless rather close together. The values of the lateral forces in Fig. 13 are very small, which is actually intended by this specific running gear design. Therefore, the measurement tolerance of $0.25 \mathrm{~N}$ has to be considered when these results are assessed.

So far we are not able to measure the slip with sufficient accuracy. Therefore, the validation of the dependency of the forces on the slip is not possible today but will be tackled soon.

\section{Conclusions and Outlook}

From an intense literature review it has been concluded that a rigid elliptical single point contact with tangential force law according to Polach is expected to provide a well balanced compromise between accuracy and computational effort in order to establish a Modelica model of the wheel-rail contact.

Therefore the related theory has been summarized in Sec. 2. One additional refinement namely the quasi-elastic instead of the pure rigid contact

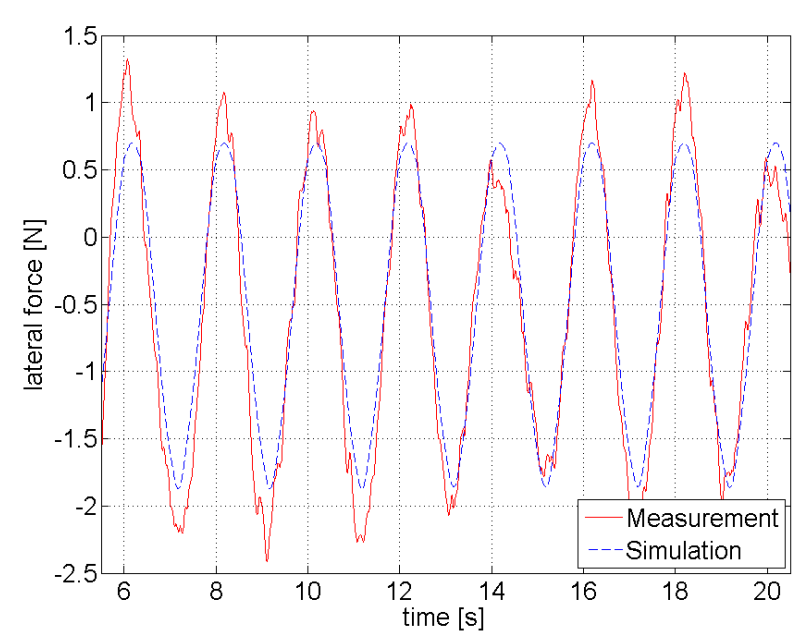

Figure 13: Comparison of lateral wheel-rail force, $v_{0}=6 \mathrm{~m} / \mathrm{s}, 0.5 \mathrm{~Hz}$ hunting frequency.

model has been introduced to guarantee a sufficient differentiability of the constraint equation.

This new Modelica wheel-rail contact is then applied to simulate the behavior of an experimental running gear on the scaled roller rig of DLR. The measured forces show a good agreement to the simulation results. A necessary advancement concerns the availability of slip measurements. Full scale applications with standard wheel-rail profiles will be modeled in the near future.

The new DLR RailwayDynamics Library, to which this wheel-rail contact model contributes a first cornerstone, is mainly intended to support the advanced observer and control design development and to facilitate multidisciplinary simulation tasks such as the interaction of running dynamics and drive train.

\section{Acknowledgements}

This work was supported by BMBF (BMBF Förderkennzeichen: 01IS12022G), the German Federal Ministry of the Education and Research, within the ITEA 2 project Modrio.

\section{References}

[1] K. Knothe and S. Stichel. Schienenfahrzeugdynamik. Springer, Berlin, 2003.

[2] Ingo Kaiser. Refining the modelling of vehicle-track interaction. Vehicle System Dynamics, 50(sup1):229-243, 2012. 
[3] O. Polach. Characteristic parameters of nonlinear wheel/rail contact geometry. Vehicle System Dynamics, 48(S1):19-36, 2010.

[4] H. Netter, G. Schupp, W. Rulka, and K. Schroeder. New aspects of contact modelling and validation within multibody system simulation of railway vehicles. Vehicle System Dynamics, 29(S1):246-269, 1998.

[5] M. Arnold and H. Netter. Wear profiles and the dynamical simulation of wheel-rail systems. Progress in Industrial Mathematics at ECMI, 96:77-84, 1997.

[6] G. Schupp, C. Weidemann, and L. Mauer. Modelling the contact between wheel and rail within multibody system simulation. Vehicle System Dynamics, 41(5):349-364, 2004.

[7] J. Piotrowski and H. Chollet. Wheel-rail contact models for vehicle system dynamics including multi-point contact. Vehicle System Dynamics, 43(6-7):455-483, 2005.

[8] J. Kalker. Three-dimensional elastic bodies in rolling contact, volume 2. Springer, 1990.

[9] J. Piotrowski and W. Kik. A simplified model of wheel/rail contact mechanics for non-hertzian problems and its application in rail vehicle dynamic simulations. Vehicle System Dynamics, 46(1-2):27-48, 2008.

[10] S. Iwnicki. Handbook of railway vehicle $d y$ namics. CRC Press, 2006.

[11] K. Knothe, R. Wille, and B.W. Zastrau. Advanced contact mechanics: Road and rail. Vehicle System Dynamics, 35(4-5):361-407, 2001.

[12] J. Kalker. A fast algorithm for the simplified theory of rolling contact. Vehicle System Dynamics, 11(1):1-13, 1982.

[13] K. Knothe. History of wheel/rail contact mechanics: from Redtenbacher to Kalker. Vehicle System Dynamics, 46(1-2):9-26, 2008.

[14] O. Polach. A fast wheel-rail forces calculation computer code. Vehicle System Dynamics, 33:728-739, 2000.

[15] O. Polach. Creep forces in simulations of traction vehicles running on adhesion limit. Wear, 258(7):992-1000, 2005.
[16] E.A.H. Vollebregt, S. Iwnicki, G. Xie, and P. Shackleton. Assessing the accuracy of different simplified frictional rolling contact algorithms. Vehicle System Dynamics, 50(1):117, 2012.

[17] J. Winter, E. Mittelbach, and J. Schykowski, editors. RTR Special - Next Generation Train. Eurailpress, DVV Media Group, 2011.

[18] M. Arnold and H. Netter. Approximation of contact geometry in the dynamical simulation of wheel-rail. Mathematical and Computer Modelling of Dynamical Systems, 4(2):162184, 1998.

[19] H. Netter. Rad-Schiene Systeme in differential-algebraischer Darstellung. Number 352 in VDI-Fortschrittsberichte Reihe 12. VDI-Verlag, Düsseldorf, 1998.

[20] W. Kortüm and P. Lugner. Systemdynamik und Regelung von Fahrzeugen. SpringerVerlag, Berlin, 1993.

[21] K. Popp and W. Schiehlen. Ground Vehicle Dynamics. Springer, 2010. 